\title{
Combining SFBC_OFDM Systems with SVD Assisted Multiuser Transmitter and Multi user Detector
}

\author{
Ahmed. Boudaa ${ }^{1}$, Merahi. Bouziani $^{2}$

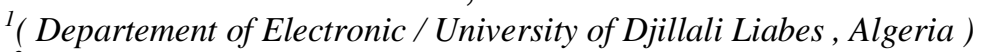 \\ ${ }^{2}$ (Departement of Electronic / University of Djillali Liabes , Algeria)
}

\begin{abstract}
In this work, we exploit the SVD assisted multiuser transmitter (MUT) and multiuser detector $(M U D)$ technique, using downlink (DL) preprocessing transmitter and DL postprocessing receiver matrice. .In combination with space frequency block coding (SFBC). And also propose the precoded DL transmission scheme, were the both proposed schemes take advantage of the channel state information (CSI) of all users at the base station (BS), but only of the mobile station (MS)'s own CSI, to decompose the MU MIMO channels into parallel single input single output (SISO), these two proposed schemes are compared to the vertical layered space time $\left(V_{-} B L A S T\right)$ combined with SFBC (SFBC_VBLAST). Our Simulation results show that the performance of the proposed scheme with $D L$ Zero Forcing $(Z F)$ transmitter for interference canceller outperforms the SFBC_VBLAST and the precoded $D L$ schemes with $Z F$ receiver in frequency selective fading channels.

Keywords - Post processing, Preprocessing,, SFBC, SVD, ZF.
\end{abstract}

\section{INTRODUCTION}

Multiple Input Multiple Output (MIMO) is a new technology that can dramatically increase the spectral efficiency by using antenna arrays at both transmitter and receiver. An effective and practical way to approaching the capacity of MIMO wireless channels is to employ space time coding (STC). There are various approaches in coding structures including space time trellis codes (STTC) [1], space time block codes (STBC) [2], space time turbo trellis codes and layered space time (LST) codes. The pure example of LST is vertical Bell laboratories space time (VBLAST) [3].

STBC is a simple and attractive space time coding scheme that was proposed by Alamouti [4]. It requires only a small degree of additional complexity. In particular, the Alamouti code offers very simple encoding, decoding and is particularly suitable for future wireless systems. Increasing demand for higher data rate requires transmission over broadband channel which is frequency selective. As a result, inter symbol interference (ISI) is introduced, which severely degrades the system performance. Using orthogonal frequency division multiplexing (OFDM) can turn frequency selective MIMO channel into a set of parallel frequency flat MIMO channels. In practice, combination OFDM and STBC is very popular and robust technique to mitigate ISI and improve the performance of the communication in terms of taking advantage of the spatial diversity in MIMO transmitting systems [5-6]. Alamouti is remarkable orthogonal transmission structure can be applied in space time or space frequency domain in OFDM systems. The orthogonal designs can be applied as space time block coded (STBC_OFDM) [7], or frequency block coded (SFBC_OFDM) [8]. The efficient design of the DL transmitter is of paramount importance for the sake of achieving a high throughput (high data rates), to obtain this, it is important to find schemes that to reduce the effects of fading and explore new type of diversity, as well as to reduce the effect of multiuser interference (MUI), also referred to as multiple access interference (MAI), and inter antenna interference (IAI). Otherwise, the performance of such STBC MIMO OFDM systems may also seriously degrade in the presence of MAI due to multiuser applications. The effect of MUI can be mitigated by employing spatio temporal preprocessing at the transmitter. Consequently, the DL receiver complexity may be reduced at the advent of transmit preprocessing at the base station (BS), a technique which is also often referred as Multiuser transmission (MUT) [9]. Employing the singular value decomposition (SVD). More specifically, the spatio temporal preprocessing technique of decomposes a MIMO channel into a set of parallel single user MIMO channels [10], which facilitates the employments of well known MIMO processing technique. If the CSI associated to different mobile station (MS) is available at the BS, the use of preprocessing scheme at the transmitter BS, allows simpler MS receiver implementation and eventually better performance.

In this paper, we propose two schemes based on SVD the zero forcing transmit scheme denoted (ZF_Tx_svd) and ZF receive scheme denoted (ZF_Rx_svd) combined with the MU SFBC OFDM system. This paper is organized as follows, in section II we present the MIMO OFDM eigenmode, section III the system overview, section IV the multi user detector, describing the ZF interference canceller at the receiver and at the 
transmitter based on SVD, section V the simulation results are provided, finally in section VI we give our conclusions.

\section{MIMO OFDM EIGENMODE}

The DL of multiuser spatial multiplexing (MUSM) communication system is investigated where antenna arrays are used at both the base transceiver station (BTS) and at K mobile users. Due to the low complexity realisation at the mobile units, and the large diversity gain, linear processing Maintaining the Integrity of the specifications for MIMO system also referred to as Closed loop MIMO, where CSI is known at the transmitter, is used to multiple user and pre-cancel inter user interference. In particular, it was proven that MIMO eigenmode (EM) transmission system is optimal because MIMO capacity is maximized. MIMO EM uses the left and right eigenvectors of the channel matrix as eigenbeamformers in the receiver and the transmitter respectively to form the orthogonal spatial eigenbeams for transmission. We utilize SVD to obtain the largest eigen. In some sense, eigenbeamforming is an optimal space time processing scheme. However, it requires SVD on every subcarrier. It has been well known that SVD technique is an appropriate way to diagonalize the MIMO channel matrix.

MIMO OFDM system utilizing $N$ subcarriers with $M_{t}$ Transmit and $M_{r}$ receive antennas signalling over MIMO frequency selective fading channel, $H_{f}:\left(M_{r} \times M_{t}\right)$ is the frequency domain channel response matrix for the $f$ th subcarrier with possibly correlated coefficient, $h_{i j}{ }^{f}: C N(0,1)$ where $i=1, \ldots M_{r}$, and $j=$ $1, \ldots M_{t}$. The columns vectors of flat fading channel matrix $H_{f}$ are usually non orthogonal. However by SVD the channel matrix, $H_{f}$ can be decomposed into diagonal matrix $D^{1 / 2}$ and two unitary matrices $U$ and $V$. Assuming perfect CSI an SVD decomposition of the MIMO channels, $H_{f}$ can be written as $H_{f}=U_{f} D_{f} V_{f}{ }^{H}$. where, $V_{f}{ }^{H}$ is the transpose conjugate of the matrix $V_{f}$, the $\left(M_{r} \times M_{r}\right)$ matrix $U_{f}$ and the $\left(M_{t} \times M_{t}\right)$ matrix $V_{f}$ are unitary matrices (i.e. $U_{f} U_{f}{ }^{H}=I M_{r}$ and $\left.V_{f} V_{f}^{H}=I M_{t}\right)$, and $D$ is a $\left(M_{r} \times M_{t}\right)$ diagonal matrixes whose non zero diagonal elements $\left\{\lambda_{1}, \lambda_{2}, \ldots \lambda_{R_{H f}}\right\}$ are the real non negative singular values of $H_{f}$. We have $R_{H f}=$ $\min \left(M_{t} \times M_{r}\right)$ is the rank of the channel matrix $H_{f}$. Therefore, the channel matrix $H_{f}$ can be decomposed into orthogonal sub channel, the closed loop MIMO OFDM system can use eigenbeamforming on a tone by tone basis to transform a frequency selecting MIMO channel into a collection of $R_{H} N$ parallel subchannels.

\section{SYSTEM OVERVIEW}

We consider a downlink multiuser environment with a BS communication supporting $K$ user mobile station (MS's) as shown in Figure. 1, the base station employs $M$ transmit antennas and the $k$ th users MS could be equipped with $R_{k}$ receive antennas as shown in Figure. 2.a and Figure. 2.b, where $=1,2, \ldots, K$ is the spatial multiplexer of the $k$ th data branch generates a $T_{k}$ dimensional vector symbol streams $S_{k}=\left[x_{k 1}(1), x_{k 2}(2), \ldots x_{k T_{k}}(n)\right]^{T}$, where $T_{k}=2$, is the transmit Alamouti matrix and $n$ is the number of the transmitted symbols stream for each user before encoding, in space frequency coding (SFC), where $n=1 . . N / 2$, ( $N=$ number of OFDM subcarrier). Where symbols $S_{k, d}(k=1,2, \ldots, K ; d=1,2)$ are chosen from the same constellation set $S$. For convenience we assume no error correction coding and a uniform allocation of power across the substreams for each user. At the BS before the SFBC encoding we implement the eigenbeamforming, preprocessing spatial multiplexing units requires CSI, and in the case of OFDM systems the transmitter requires preprocessing knowledge for all subcarriers, the symbol vectors $n$ for the $k$ th user is multiplied by a $\left(M \times T_{k}\right)$ preprocessing matrix $P_{k}$ as shown in Figure. 1, yielding:

$$
X_{k}=P_{k} S_{k} \quad=1,2, \ldots, K
$$

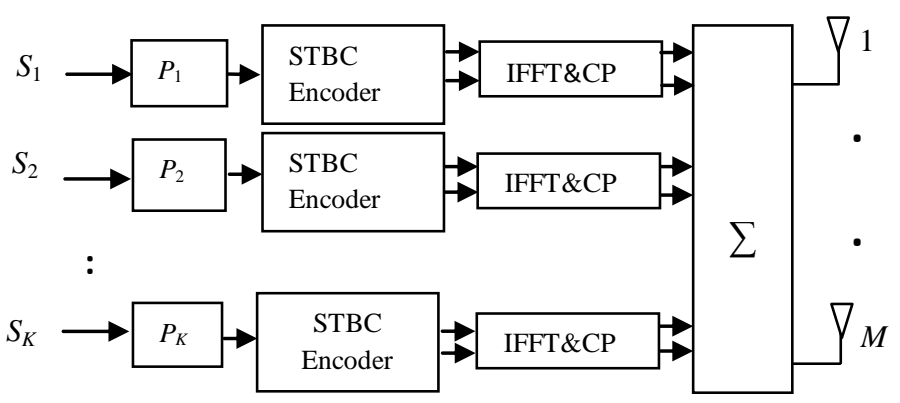

Figure. 1. Block diagram of the DL MUT Preprocessing

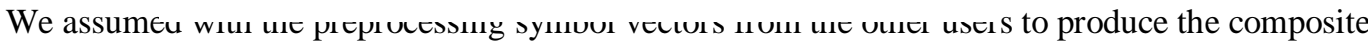
transmitted symbol vector. 


$$
X_{k}=\sum_{k=1}^{K} P_{k} S_{k}
$$

The preprocessed symbol vectors are passed through the combining block SFBC_OFDM encoder, by using the Alamouti schemes in frequency coding. A block of data symbols (OFDM symbol) transmitted over each transmitter passes through an $N$ point inverse fast Fourier transform (IFFT), and the cyclic prefix (CP) is appended. The BS transmitter broadcast the signal to multiple $K$ users simultaneously over the same frequency band through the frequency selective fading channel. At each receiver as shown in Figure. 2.a and Figure. 2.b, the CP removed and the fast Fourier transform (FFT) is applied to revert the received signals back to frequency domain. Hence the frequency selective MIMO channel is decoupled into $N$ parallel flat fading channels. The $R_{k}$ dimensional received signal $y_{k}$ at the $k$ th user where $R_{k}=1,2$ is a superposition of the $K$ signal branches distorted by channel fading plus additive white Gaussian noise (AWGN).

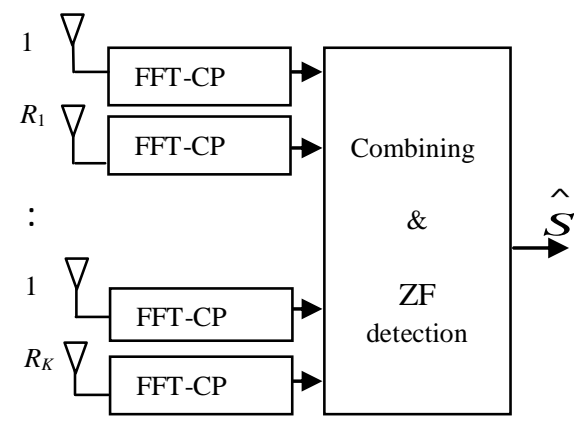

Figure. 2.a. Block diagram of the DL ZF receiver

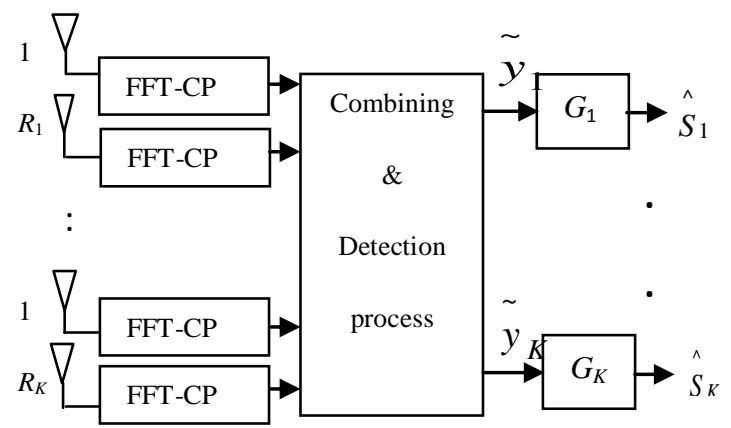

Figure. 2.b. Block diagram of the DL MUD

The complex baseband representation of the received signal vectors in the $f$ th subcarrier where $f=1 \ldots N$ can be expressed as: $y_{k}=\sum_{j=1}^{K} H_{k} C_{j}+w_{k}$

The component $C$ is the code matrix of Alamouti scheme of the OFDM symbols. The channel transfer matrix between the BS and the $k$ th user $H_{k}:\left(R_{k} \mathrm{x} M\right)$ is the frequency domain channel response matrix for subcarrier $f$, and $h_{r, m}^{k}$ is the fading coefficient modelled as a finite impulse response filter (FIR) with $L$ taps, associated with the $m$ th BS antenna and $r$ th receive antenna of user $k$, at a given subcarrier $f$.

Let $\quad h_{r m}^{k}=\left[h_{r m}^{k}(0), h_{r m}^{k}(1), \ldots . h_{r m}^{k}(L-1)\right]$

Where $r=\left\{1, \ldots, R_{k}\right\}$ and $m=\{1, \ldots \ldots M\}$. The elements of $H_{k}$ are samples of independent and identically distributed (i,i,d) complex Gaussian random variables, circularly symmetric distributed with unit variance $C N(0,1)$. The additive white Gaussian noise (AWGN) at the $r$ th receive antenna of user $k, w=\left[w_{1}, w_{1}, \ldots, w_{R_{k}}\right]$ follows distribution $C N\left(0, \sigma_{w}{ }^{2} I\right)$, where $I$ is the $\left(R_{k} \times R_{k}\right)$ identity matrix. Finally the entire multiuser MIMO channel may be characterized by the supermatrix $H$, which may be constructed by concatenating the corresponding channel matrices $\left\{H_{k}\right\}_{k=1}^{K}$ associated with each of the MS's, and the composite channel matrix is denote as:

$H=\left[H^{(1)}, H^{(2)}, \ldots . H^{(K)}\right]^{T}$

We can write the equation (3), hence the signal form become as follows:

$$
\begin{gathered}
\tilde{y}_{k}=\widetilde{H}_{k} P S+w_{k}=\widetilde{H}_{k} P_{k} S_{k}+\sum_{j=1, j \neq k}^{K} \widetilde{H}_{k} P_{j} S_{j}+w_{k} \\
=\widetilde{H}_{k} P_{k} S_{k}+Z_{k}+w_{k} \quad k=1,2, \ldots, K
\end{gathered}
$$

$P_{k}$ and $S_{k}$ is the preproceesing matrix and the transmitted symbols vector $n$ corresponding to the $k$ th user. From

(6) we see that the MUI component on the $k$ th user is represented as $Z_{k}$, and $\widetilde{H}_{k}$ is the equivalent channel transfer matrix between the BS and the $k$ th user .If we have CSI available at both the transmitter and the receiver, and then we use the SVD decomposition of the channel user $\widetilde{H}_{k}[11]$.

$\widetilde{H}_{k}=U_{k}\left[D_{k}{ }^{\frac{1}{2}}, 0\right] V_{k}=U_{k}\left[D_{k}{ }^{\frac{1}{2}}, 0\right]\left[\begin{array}{c}V_{k s}{ }^{H} \\ V_{k n}{ }^{H}\end{array}\right]$

Where $U_{k}$ and $V_{k}$ are $\left(2 R_{k} \times 2 R_{k}\right)$ and $(M \times M)$ component unitary matrices, respectively, and $D_{k}$ is an $\left(2 R_{k} \times 2 R_{k}\right)$ component diagonal matrix containing the eigenvalues of $\widetilde{H}_{k} \widetilde{H}_{k}{ }^{H}$.

IV.

\subsection{ZF Receive Scheme Based On SVD}

\section{Multi User Detector}


The optimal eignbeamforming as shown in [12-13], is the precoding matrix where $P=V$, which is derived from (7) and consequently the precoding matrix is defined as:

$$
P_{k}=V_{k} \quad k=1,2, \ldots, K
$$

By substituting (8) in (6) the received signal can be expressed as:

$\tilde{y}_{k}=\widetilde{H}_{k} V_{k} S_{k}+Z_{k}+w_{k}$

$V_{k}$ and $S_{k}$ is the precoding matrix and the transmitted symbols vector $n$ corresponding to the $k$ th user, and the equivalent effective channel can be expressed by:

$\widetilde{H}=\left[\widetilde{H}_{1}, \widetilde{H}_{2}, \ldots, \widetilde{H}_{K}\right]^{T}$

The composite multiuser received signal may be express as:

$\tilde{y}_{k}=\widetilde{H} V S+w$

$V=\left[V^{(1)}, V^{(2)}, \ldots V^{(K)}\right], w=\left[w^{(1)}, w^{(2)}, \ldots, w^{(K)}\right]$

$V$ and $w$ are the space time precoding supermatrix, as well as the AWGN noise. The receiver sees the equivalent channel matrix.

$$
\widetilde{\widetilde{H}}=\widetilde{H} V
$$

And according to Figure. 2.a we can detect the desired signal vector streams $S$ by the implementation of the $\mathrm{ZF}$ detection of equivalent channel matrix, hence remove the interference between the transmitted user streams and subsequently implemented the SFBC decoding. With the precoding matrix and the equivalent effective channel, we can express the ZF receiver based on SVD solution which is referred to ZF_Rx_svd as follow:

$\widetilde{\widetilde{H}}_{Z F}=V^{H} \widetilde{H}_{Z F}$, where $\widetilde{H}_{Z F}=\left(\widetilde{H}^{H} \widetilde{H}^{-1} \widetilde{H}^{H}\right.$

4.1. ZF Transmit Scheme Based On SVD

In this section we present the ZF transmit scheme to pre-cancel the MUI interference based on the SVD, at the BS station, from (6) and (7) the received signal of the $k$ th user $\tilde{y}_{k}$ may be expressed as:

$\tilde{y}_{k}=U_{k} D^{1 / 2} V_{k s}{ }^{H} P S+w_{k} \quad k=1,2, \ldots, K$

Then the overall DL received signal vector streams $\tilde{y}_{k}$ of the $K$ M's can be expressed as:

$\tilde{y}_{k}=U D^{1 / 2} V_{s}^{H} P S+w$

According to [11], The DL BS transmit preprocessing matrix $P$ is designed, that the DL MUI can efficiently be suppressed. The MUI can fully be removed when the DL preprocessing matrix to satisfy:

$P=\left[V_{s}^{H}\right]^{+} B=\bar{P} B$

(16)

Where the power allocation regime of $B=\operatorname{diag}\left\{B_{11}, B_{1 T_{k}} ; \ldots, B_{K 1}, \ldots, B_{K T_{k}}\right\}$ and $\left[V_{s}^{H}\right]^{+}$denotes the pseudo inverse of the matrix $V_{s}^{H}$, which is referred to ZF_Tx_svd, and $\bar{P}=V_{s}\left[V_{s}^{H} V_{s}\right]$.

When substituting the overall DL preprocessing matrix of (16) into (15) the overall received signal vector $y$ of $K$ MS user can be simplified to

$\tilde{y}_{k}=U D^{1 / 2} B S+w$

The symbol vector of the $k$ th MS can be expressed as:

$\tilde{y}_{k}=U_{k} D_{k}^{1 / 2} B_{k} S_{k}+w_{k}$

The IAI can be suppressed with the SVD based matrices $\left\{U_{k}\right\}$ of (7). By $\left\{G_{k}=U_{k}{ }^{H}\right\}$ according to Figure. 2.b the user specific decision variables can individually be expressed as:

$\hat{S}_{k}=D_{k}{ }^{1 / 2} B_{k} S_{k}+U_{k}{ }^{H} w_{k} \quad k=1,2, \ldots, K$

And the overall decision variables can expressed as:

$$
\hat{S}=D^{1 / 2} B S+U^{H} w
$$

The overall of the user decision symbol stream can be expressed as: $\hat{S}=\left[\hat{S}_{1}, \hat{S}_{2}, \ldots \ldots, \hat{S}_{K}\right]^{T}$

\section{Simulation Results}

In this section, we consider SFBC system with $N=64$ ( $N$ : number of subcarrier OFDM). Combined with the SVD MUT and MUD by applying the ZF receiver and ZF transmitter scheme and compared them with ZF_VBLAST. The maximum channel delay spread and the CP length are the same and equal to $L=16$. In other words, channel with long excess delay time, exhibit a lower coherence bandwidth corresponding to higher frequency selectivity than shorter channel. The SISO channels from the transmit antennas to the receive antennas are assumed to be independent and Rayleigh distributed. The complex symbols are assumed to be quaternary phase shift keying (QPSK).

Figure.3. Shows the BER performance comparison of two users SFBC_OFDM system. When each user has one receive antenna and 4 BTS transmit antennas $(4 \times 2$ users/1Rx). It is shown that the ZF_Tx_sdv scheme's outperforms the ZF_VBLAST by $16 \mathrm{~dB}$, and the ZF_Rx_svd by $20 \mathrm{db}$ at BER=10 $0^{-4}$. VBLAST, decodes the substreams, subtracts its interference and then decodes the later substreams. However, was not 
designed for exploiting transmits diversity. The performance of VBLAST receiver is mainly dependent on the first substreams, which has the lowest diversity gain and limited by error propagation. Where in ZF_Rx_svd, the noise term may be amplified in a way that influences the decoding output in negative way.

Figure.4. Shows the BER performance comparison of two users SFBC_OFDM system. When each user has two receive antenna and 4 BTS transmit antennas ( $4 \times 2$ users $/ 2 \mathrm{Rx})$.

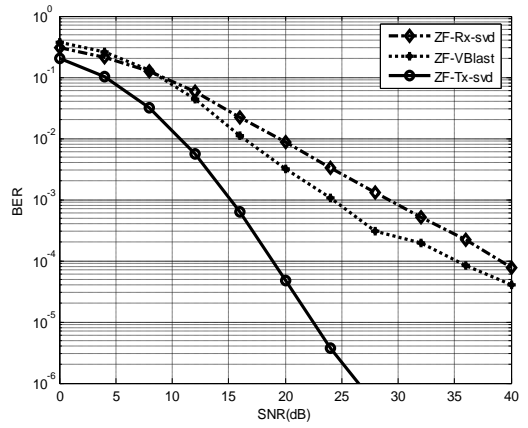

Figure.3. BER performance comparison of 2 users SFBC_OFDM System, with 4 BTS transmit antenna and 1 receive antenna per user, using $\mathrm{ZF}_{-}$Tx_svd

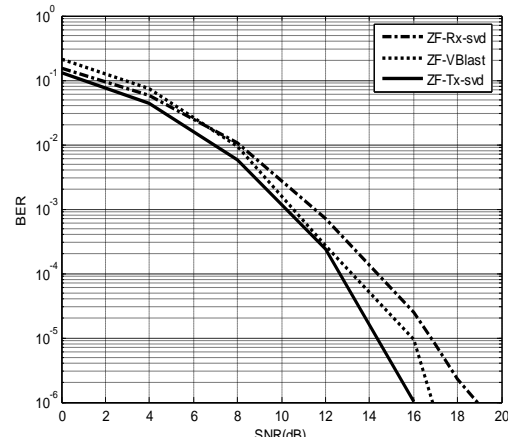

Figure.4. BER performance comparison of 2 users SFBC_OFDM System, with 4 BTS transmit antenna and 2 receive antenna per user, using ZF_Tx_svd

It is shown that the ZF_Tx_svd scheme's outperforms the ZF_VBLAST by $1 \mathrm{~dB}$, and the ZF_Rx_svd by $3 \mathrm{db}$ at $\mathrm{BER}=10^{-6}$. By adding one antenna at each receiver, however, a significant SNR reduction of $10 \mathrm{~dB}$ is achieved at $\mathrm{BER}=10^{-6}$ for $\mathrm{ZF}_{-} \mathrm{Tx} \_$svd scheme. The eigenmode selection achieves a higher diversity order than simple spatial multiplexing and increase the diversity gain.

\section{CONCLUSION}

Multiuser SFBC_OFDM system combined with SVD assisted MUT and MUD is studied in the frequency selecting fading channel, from the results the ZF transmit scheme based on SVD for MUI precanceller outperforms the ZF_VBLAST and ZF receiver based on SVD. It can be concluded that the space frequency coded system coupled with SVD assisted MUT and MUD with ZF transmit scheme efficiently exploits diversity techniques. These are, space and frequency diversity to overcome fading found in the radio channel, and provides high performance.

\section{References}

[1] V. Tarokh, N. Seshadri, and A. R. Calderbank, Space-time codes for high data rate wireless communication, Performance criterion and code construction, IEEE Trans. Inform. Theory, vol.44, no.2, 744-765, Mar.1998.

[2] V. Tarokh, H. Jafarkhani and A .R. Calderbank, Space-time block codes from orthogonal design, IEEE Trans. Info. Th., vol. 45, no. 5, 1456-1467, Jul. 1999.

[3] Thuong Le-Tien, Tuan Ly-Huu, V BLAST Detection in Space Time Coding Wireless system and implementation on FPGA, International Symposium on Electrical Electronics Engineering 2007-Oct 24, 25 2007-HCM City, Vietnam .

[4] S.M. Alamouti. A simple transmit diversity technique for wireless communications. IEEE J.Select. Areas Common, vol.16, 14511458.1998.

[5] A.Doufexi, E.Tameh, A.Nix, A.Pal, M.Beach, C.Williams, Throughput and Coverage of WLANs Employing STBC under Different Channel Conditions, ISWCS 2004, Mauritius, September 2004.

[6] Muhammad Imadur Rahman, Nicola Marchetti, Suvra Sekhar Das, Frank H.P. Fitzek, Ramjee Prasad, Combining Orthogonal Space Frequency Block Coding and Spatial Multiplexing in MIMO-OFDM System,"'(is available at http:// www.kom.aau.dk/ ff/document/ InOWo 2005).

[7] K. F. Lee and D. B. Williams, A space-time coded transmitter diversity technique for frequency selective fading channels, in Proc. IEEE Sensor Array and Multichannel Signal Processing Workshop, Cambridge, MA, 149-152. March 2000.

[8] K. F. Lee and D. B. Williams, A Space-frequency transmitter diversity technique for OFDM systems, in Proc. IEEE GLOBECOM, San Francisco, CA, 1473-1477,Nov. 2000.

[9] R.Irmer, Multiuser transmission in code division multiple access mobile Communications systems, Ph.D. dissertation, Technische Universitat Dresden, 2005.

[10] Gordon L. Stuber, John Barry, Steve W. Mc Laughlin,Ye (Geoffrey) Li, Mary Ann Ingram, Thomas G. Broadband MIMO OFDM Wireless Communications, "(is available at http:// www.ece.gatech.edu/ users/stuber/nsf3/ pubs/./proc-ieee.).

[11] W.Liu, L. L.Yang, and L. Hanzo, SVD assisted multiuser transmitter and multiuser detector design for MIMO system, IEEE Transactions on Vehicular Technology, Vol. 58, No. 2, February 2009.

[12] Daniel Schneider, Joachim Speidel othar Stadelmeier, Dietmar Schill, Precoded Spatial Multiplexing MIMO for Inhome Power Line communications, in Global Telecommunications Conference (IEEE GLOBECOM 2008), New Orleans, 2008.

[13] Runhua Chen, Robert W. Heath, Jeffrey G. Andrews, Transmit Selection Diversity for Unitary Precoded Multiuser Spatial Multiplexing Systems With Linear Receivers, proc of IEEE Global Telecommunications Conference, Nov. 29-Dec. 3, 2004, Dallas, TX, USA. (is available at http:// www.users. ece.utexas.edu/ rheath/ papers/ 2005/. 